\title{
Autism and Beauty: Neural Correlates of Aesthetic Experiences in Autism Spectrum Disorder
}

\author{
Seong Kyoung Park', Jung-Woo Son², Seungwon Chung ${ }^{3}$, Seungbok Lee ${ }^{4}$, Hei-Rhee Ghim ${ }^{4}$, \\ Sang-Ick Lee ${ }^{2}$, Chul-Jin Shin ${ }^{2}$, Siekyeong $\mathrm{Kim}^{2}$, Gawon Ju${ }^{1}$, Sang Cheol Choi ${ }^{5}$, \\ Yang Yeol Kim ${ }^{6}$, Young Jin $\mathrm{Koo}^{7}$, Bung-Nyun $\mathrm{Kim}^{8}$, and Hee Jeong $\mathrm{Yoo}^{9}$ \\ ${ }^{1}$ Department of Psychiatry, Chungbuk National University Hospital, Cheongju, Korea \\ ${ }^{2}$ Department of Neuropsychiatry, College of Medicine, Chungbuk National University, Cheongju, Korea \\ ${ }^{3}$ Department of Psychiatry, Bugok National Hospital, Changnyeong, Korea \\ ${ }^{4}$ Department of Psychology, Chungbuk National University, Cheongju, Korea \\ ${ }^{5}$ Didim Psychiatric Clinic, Seoul, Korea \\ ${ }^{6}$ SeoulTop Child Adolescent Psychiatric Clinic, Seoul, Korea \\ ${ }^{7}$ MindDoctor's Clinic for Junior, Seoul, Korea \\ ${ }^{8}$ Division of Child and Adolescent Psychiatry, Department of Psychiatry, Seoul National University College of Medicine, Seoul, Korea \\ ${ }^{9}$ Division of Child and Adolescent Psychiatry, Department of Psychiatry, Seoul National University Bundang Hospital, \\ Seoul National University College of Medicine, Seongnam, Korea
}

Objectives: The purpose of this study was to investigate whether the neural activity of autism spectrum disorder (ASD) patients is different from that of normal individuals when performing aesthetic judgments.

Methods: We recruited typical ASD patients without savant skills (ASD group, $n=17$ ) and healthy controls (HC group, $n=19)$ for an functional magnetic resonance imaging study. All subjects were scanned while performing aesthetic judgment tasks on two kinds of artwork (magnificent landscape images and fractal images). Differences in brain activation between the two groups were assessed by contrasting neural activity during the tasks.

Results: The aesthetic judgment score for all images was significantly lower in the ASD group than in the HC group. During the aesthetic judgment tasks, the ASD group showed less activation than the HC group in the anterior region of the superior frontal gyrus, and more activation in the temporoparietal area and insula, regardless of the type of images being judged. In addition, during the aesthetic judgment task for the fractal images, the ASD group exhibited greater neural activity in the amygdala and the posterior region of the middle/inferior temporal gyrus (Brodmann area 37) than the HC group.

Conclusion: The results of this study suggest that the brain activation patterns associated with aesthetic experiences in ASD patients may differ from those of normal individuals.

Key Words: Autism spectrum disorder; Beauty; Aesthetic experience; Neuroaesthetics; Brain; Functional magnetic resonance imaging.

Received: October 12, 2017 / Revision: January 25, 2018 / Accepted: February 14, 2018

Address for correspondence: Jung-Woo Son, Department of Neuropsychiatry, College of Medicine, Chungbuk National University, 1 Chungdae-ro, Seowon-gu, Cheongju 28644, Korea

Tel: +82-43-269-6187, Fax: +82-43-267-7951, E-mail: mammosss@hanmail.net

\section{INTRODUCTION}

Autism spectrum disorder (ASD) is a chronic disease that is characterized by communication and social interaction disabilities, as well as by the presence of repetitive and stereotyped behaviors. ${ }^{1)}$ Since these symptoms begin at infancy and early childhood, the psychological distress and burden to ASD patients and their family members are severe, and considerable socioeconomic costs are required for their treat-

This is an Open Access article distributed under the terms of the Creative Commons Attribution Non-Commercial License (http://creativecommons.org/licenses/by-nc/4.0) which permits unrestricted non-commercial use, distribution, and reproduction in any medium, provided the original work is properly cited. ment. Moreover, although various treatment methods have been attempted, a lack of treatments leading to marked improvements in the autistic state persists, likely due to the fact that the disabilities demonstrated by ASD patients appear across all domains of cognition, affect, and behavior.

Despite this, several research have recently shown that not all functions are reduced in ASD patients, and that some ASD patients can perform certain functions extraordinarily well. ${ }^{2,3)}$ ASD patients who exhibit superior abilities are referred to as "savants." The arts field, such as visual arts and music, is one particular field that has received attention in this regard. For example, Glenn Gould, a globally renowned 
pianist from Canada, showed various psychological and behavioral characteristics that pertained to ASD. ${ }^{4)}$ Stephan Wiltshire, a famous autistic artist from England, was referred to as the "human camera" because he drew the urban landscapes of New York, Tokyo, and other major cities very precisely, down to the finest details, after a single helicopter ride. ${ }^{5}$ )

There have been many psychological and neuroscientific attempts to explain the extraordinary abilities of such ASD patients in specific artistic fields. Boso et al. ${ }^{4}$ explained these abilities with a theory of "weak central coherence. ${ }^{96,7)}$ That is, rather than connection impairments among areas in the brain causing weak central coherence, "domain-specific" knowledge may increase excessively, leading to outstanding abilities. Wallace et $\mathrm{al}^{8}{ }^{8}$ scanned the brain of an autistic patient with ingenious talents for drawing using magnetic resonance imaging (MRI), and found that cortical thickness was significantly lower in the medial prefrontal area, premotor area, and middle temporal area, while cortical thickness of the superior parietal lobule was significantly higher than that of normal individuals. They argued that the results of their study could explain the lower social cognitive abilities and higher visuospatial abilities of the patient.

There have been many efforts to promote the social rehabilitation of ASD patients with superior artistic abilities through the use of their talents. A social enterprise in Korea offers a design school for autistic patients, and recruits them into other professional design companies. ${ }^{9}$ Efforts have also been made to improve the sociability of autistic patients through music or art education.

When typical ASD patients who do not demonstrate superior artistic abilities have an aesthetic experience through creating or appreciating aesthetic works, a question remains regarding whether their aesthetic judgments are similar to those of normal individuals, and whether brain activities related to aesthetic judgments differ from those of normal individuals when aesthetic judgments are made. Research addressing these questions has not yet been reported.

"Neuroaesthetics" refers to the research field that explores the brain mechanisms underlying aesthetic experiences. Neuroaesthetics focuses on changes that occur in the brain during aesthetic experiences, or when making aesthetic judgments, through neuroscientific methods, and is currently a tremendously popular field. ${ }^{5,10)}$

For example, Cupchik et al. ${ }^{11)}$ reported that aesthetic experiences were related to the activation of brain areas related to emotion, such as the bilateral insula. Kawabata and Zeki ${ }^{12)}$ examined normal subjects who were asked to judge various paintings as "beautiful," "neutral," or "ugly," and found increased activation in the orbitofrontal cortex for paintings that were judged to be "beautiful," and decreased activation in the orbitofrontal cortex and increased activation in the motor cortex for paintings that were deemed "ugly."

The medial frontal area around Brodmann areas (BA) 9 and 10 and the ventral prefrontal cortex around BA 45/47 are also known to be activated during aesthetic experiences. There are also reports that the medial frontal area is activated simultaneously with the posterior cingulate gyrus or precuneus. Whether these areas are activated specifically for aesthetic judgments or whether they are areas that are generally activated for tasks involving social judgment is currently under considerable debate. ${ }^{13,14)}$ Vartanian and Goel ${ }^{15)}$ argued that the activation of the anterior cingulate gyrus and caudate nucleus changes according to changes in preferences for pictures, suggesting that aesthetic experiences are related to activation of the brain's reward system.

Son et al. ${ }^{5)}$ organized topics related to neuroaesthetic research into six categories: 1) aesthetic experiences and the brain's reward system, 2) embodiment, 3) differences in brain activation between aesthetic and pragmatic views, 4) differences in aesthetic experiences according to the type of artwork expression, 5) aesthetic emotion, and 6) sublime. Further, $\mathrm{Kim}^{16)}$ emphasized that various contextual effects that control aesthetic experience must be taken into account in neuroaesthetic research, and that the number of reports on changes in the activation of the brain's default mode network during aesthetic experience is increasing in recent neuroaesthetic research studies.

In the present study, we aimed to examine whether typical ASD patients without superior artistic abilities show different characteristics from normal individuals when making aesthetic judgments on artwork, and whether there are differences in brain activation between typical ASD patients and normal individuals when making such aesthetic judgments, using functional magnetic resonance imaging (fMRI). Moreover, in order to determine whether there are differences in aesthetic judgment and brain activation between the two groups when the type of artwork differs, we selected two types of artwork (magnificent landscape images and fractal images) for this study.

\section{METHODS}

\section{Subjects}

The subjects in the ASD group were recruited through child and adolescent psychiatric clinics in Seoul and Cheongju, while the subjects in the control group were recruited by use of advertisements in general psychiatric clinics, high schools, colleges, and academies located in Seoul. The criteria that were used for both groups were as follows: 1) an age of 15-22 years, 2) IQ of 70 or higher based on the results of the short 
form intelligence quotient test, 3) no previous private art education (aside from regular art education at school) or previous education from an art academy (college students must not be majoring in the arts field), 4) no history of brain injury or seizure disorders, and 5) no severe medical conditions. In addition, subjects in the ASD group were diagnosed with ASD by psychiatrists who had completed child and adolescent psychiatry fellowships, and in accordance with Diagnostic and Statistical Manual of Mental Disorders-5 (DSM5). In addition, they did not meet criteria for major mental disorders, based on the Kiddie-Schedule for Affective Disorders and Schizophrenia-Present and Lifetime Version-Korean Version (K-SADS-PL-K), ${ }^{1718)}$ which is a semi-structured interview for diagnosing mental illnesses in children and adolescents. The subjects in the control group had no history of mental disorders, did not satisfy the criteria for the DSM-5 diagnoses for ASD, and also did not meet the criteria for any diagnoses based on the K-SADS-PL-K. All subjects completed the short form intelligence quotient tests, KSADS-PL-K, and all assessments of psychological characteristics used in this study. Only the subjects who were not disqualified due to head movement and also completed the E-Prime Version 2.0 (Psychological Software Tools, Pittsburgh, PA, USA). tasks during the fMRI scan were included in the analysis. There were 17 subjects in the ASD group and 19 subjects in the control group for the analysis.

This study was conducted in accordance with the final version of the Declaration of Helsinki and was approved by the Bioethics Committee at Chungbuk National University Hospital (IRB No. CBNUH 2013-11-007-001). Subjects were required to complete a consent form after a thorough explanation of the purpose and methods of the study was provided, either to the subjects themselves, if they were 19 years of age or older, or to both the subjects and their parents, if they were under 19 years old.

\section{Intelligence and psychological assessment}

\section{Intelligence assessment}

The short form of the Korea Educational Development Institute Wechsler Intelligence Scale for Children was used to evaluate the intelligence of adolescent subjects in the age of 15, and four subtests were conducted, on "Similarity," "Picture Completion," "Arithmetic," and "Block Design." The short form of the Korean-Wechsler Intelligence Scale III was used to assess the intelligence of subjects 16 year or older, and two subtests were performed, on "Information" and "Picture Completion."

\section{Assessment of psychological characteristics}

Autism-Spectrum Quotient (AQ)

Autism Spectrum Quotient (AQ) is a self-report questionnaire that evaluates the autistic tendencies of an individual with normal intelligence. Higher scores imply stronger autistic tendencies. ${ }^{19)}$ This study used a questionnaire that was adapted by five psychologists who majored in psychology with at least 20 years of professional experience. ${ }^{20)}$ The questionnaire included 50 questions, each of which could be scored from 0 to 1 point, for a total score range of 0-50 points. The reliability coefficient (Cronbach's $\alpha$ ) of the questions was 0.71 .

\section{Empathy Quotient (EQ)}

Empathy Quotient (EQ) is a self-report questionnaire that measures empathy, which is the ability to understand, consider, and empathize with another person's feelings. Higher scores imply a greater level of empathy. ${ }^{21)}$ This study used a questionnaire that was adapted by five psychologists who majored in psychology with at least 20 years of experience. ${ }^{16)}$ The questionnaire includes 20 questions unrelated to empathy and 40 questions specifically testing empathy. Each question could be scored from 0 to 2 points, for a total score range of $0-80$ points. The reliability coefficient (Cronbach's $\alpha$ ) of the questions was 0.79 .

\section{Systemizing Quotient-Revised (SQ-R)}

Systemizing is the ability to understand and analyze a system that operates according to a principle and also to determine the corresponding rules. While ASD patients have reduced empathy, they are believed to have relatively strong systemizing abilities. ${ }^{22)}$ Systemizing Quotient-Revised (SQ$\mathrm{R}$ ) is a self-report questionnaire that can assess systemizing skills. Higher scores imply higher systemizing abilities. ${ }^{23)}$ Each of the 75 questions is scored from 0 to 2 points, for a total score range of $0-150$ points.

\section{fMRI implementation plan and data analysis}

\section{Experimental fMRI design}

This study was an fMRI study with a block design. The images to be used for the experimental task during the fMRI scan were collected from the internet. First, magnificent landscape images and fractal images with high resolution were selected. There is a precedence for using magnificent landscape images ${ }^{10)}$ or fractal images ${ }^{24)}$ from previous neuroaesthetic studies, and this study selected new images referred to by these studies. All images were PNG files, with the image resolution set to $640 \times 480$ so that they could be used during 


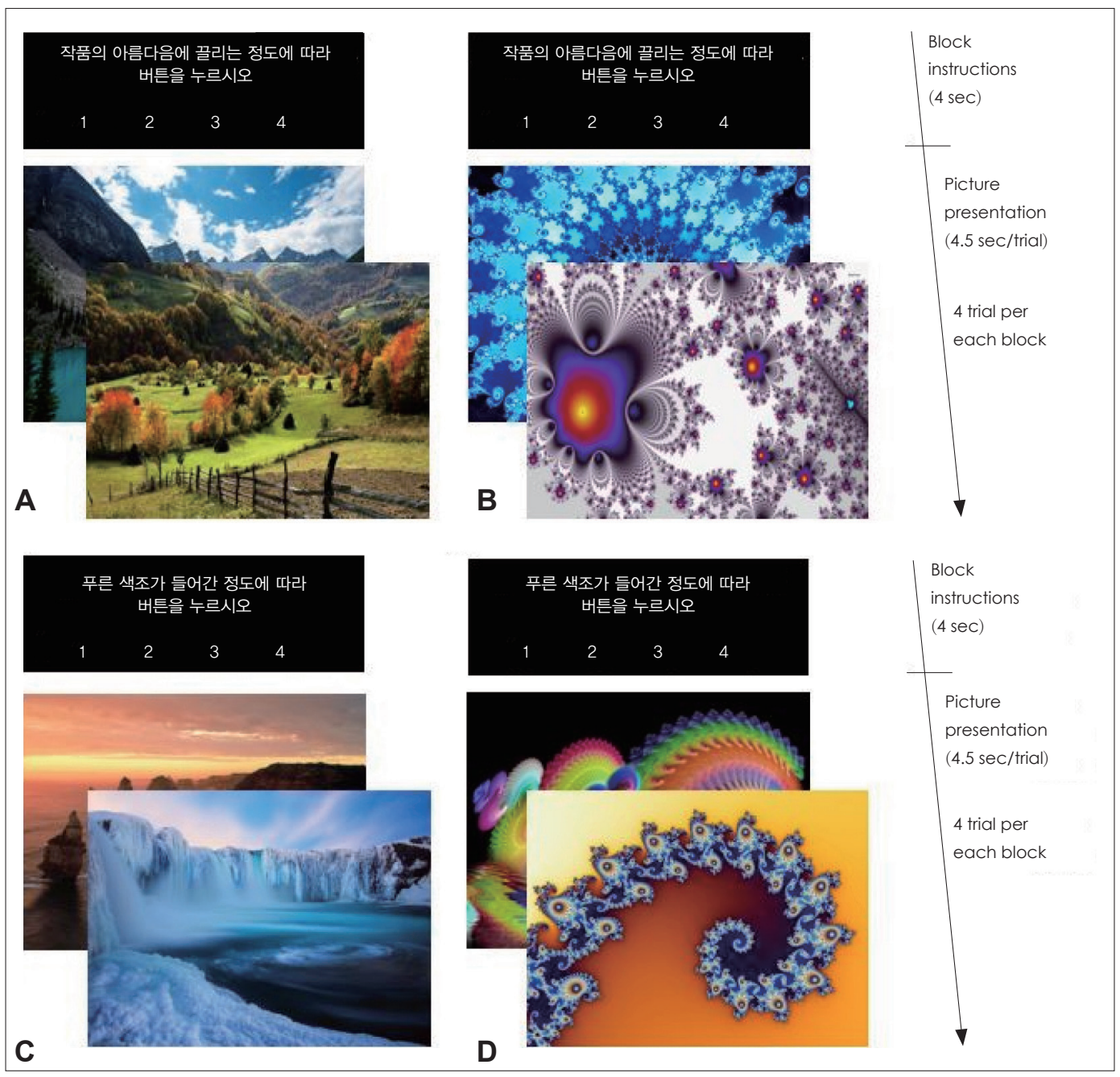

Fig. 1. Examples of (A) aesthetic-landscape task, (B) aesthetic-fractal task, (C) control-landscape task, and (D) control-fractal task. Aesthetic judgement block was introduced by a instruction "Press the button depending on how much you are attracted to the beauty of the work" and Control block was introduced by a instruction "Press the button according to the degree of blue tone" in Korean.

the fMRI scan. A total of 48 pieces of artwork were selected, including 24 magnificent landscape images and 24 fractal images (Fig. 1).

The aesthetic judgment task and the control judgment task were designed with the stimuli categorized into landscape or fractal images. Therefore, a total of four different judgment tasks were required: 1) an aesthetic judgment of the landscape images, 2) an aesthetic judgment of the fractal images, 3) a control judgment of the landscape images, and 4) a control judgment of the fractal images. Ultimately, a $2 \times 2$ factorial design was used. Each judgment task consisted of 6 blocks, for a total of 24 blocks. Each block started with a prompt for 4 seconds and then four trials were conducted, with each trial lasting 4.5 seconds. After each block, a 12-second resting period occurred. Including 6 seconds for a dummy period, the total scan time was 13 minutes and 42 seconds.

Before the fMRI scan, the subjects used a laptop computer in a waiting room outside the fMRI room to practice each task. The image stimuli that were presented during the practice were different from the stimuli that were used during the scan.

Task stimuli were presented using E-Prime Version 2.0. The details of each task condition were described below.

"Aesthetic-landscape (AL)" task

The prompt, written in Korean, stated "Press the button depending on how much you are attracted to the beauty of the work," then the landscape images were presented one by one. Subjects pressed a button signifying 1 to 4 points according to how beautiful they judged the artwork to be. 1 point meant they were least attracted to the artwork, while 4 points meant they were most attracted to it.

"Aesthetic-fractal (AF)" task

The prompt, written in Korean, stated "Press the button de- 
pending on how much you are attracted to the beauty of the work," then the fractal images were presented one by one. Subjects pressed a button signifying 1 to 4 points according to how beautiful they judged the artwork to be. 1 point meant they were least attracted to the artwork while 4 points meant they were most attracted to it.

\section{"Control-landscape (CL)" task}

The prompt, written in Korean, stated "Press the button according to the degree of blue tones," then the landscape images were presented one by one. Subjects then pressed a button signifying 1 to 4 points according to the degree of blue tones in the image; 1 point if there was $25 \%$ or less blue tones in the overall image, 2 points if the overall image was between $26-50 \%$ blue tones, 3 points if the image was between $51-75 \%$ blue tones, and 4 points if there was $75 \%$ or more blue tones in the image. Subjects were allowed to practice sufficiently before the scan so that they could become accustomed to the control judgment task.

\section{"Control-fractal (CF)" task}

The prompt, written in Korean, stated "Press the button according to the degree of blue tones," then fractal images were presented one by one. The subject then pressed a button signifying 1 to 4 points according to the degree of blue tones in the image, as described above for the landscape images. Subjects were allowed to get sufficient practice before the scan, as well, also as described above.

The visual stimuli were presented through goggles (magnet-compatible and three-dimensional goggles) worn by the subjects, and an eye tracker installed in the goggles tracked the subject's eye movements. The experimenter was able to give instructions through headphones worn by the subjects, who were instructed not to move or speak during the scan.

\section{Image acquisition}

All subjects were scanned using a Siemens 3T Tim Trio scanner (Siemens Healthcare, Erlangen, Germany) at the Brain Imaging Center at Korea University. The blood oxygen level dependent method was applied through the echo planar imaging sequence for the fMRI, and the thickness of each image was $4.0 \mathrm{~mm}$. No gap between the slices was permitted. Other MRI parameters included repetition time $(\mathrm{TR})=2000 \mathrm{~ms}$, echo time $(\mathrm{TE})=30 \mathrm{~ms}$, flip angle $=90^{\circ}$, field of view $=1440 \times$ $1440 \mathrm{~mm}$, and matrix $=64 \times 64$. In addition, the MRI parameters in the $\mathrm{T} 1$ anatomical scan were $\mathrm{TR}=1900 \mathrm{~ms}, \mathrm{TE}=2.52$ $\mathrm{ms}$, flip angle $=9^{\circ}$, field of view $=256 \times 256 \mathrm{~mm}$, and matrix $=$ $256 \times 256$.

\section{Data analysis}

The imaging data were analyzed using the SPM8 software (Wellcome Trust Centre for Neuroimaging, Institute of Neurology, UCL, London, UK), and a general linear model was applied. Motion corrections were made for each subject's brain image data, and the data was normalized in order to assess anatomical location. The kernel size was set to $8 \mathrm{~mm}$ for the smoothing process. A whole brain analysis was conducted first to survey brain regions that were activated. In the within-group and regression analyses, clusters that passed the voxel-level uncorrected threshold of $\mathrm{p}<0.0005$ and exceeded 20 voxels in size were considered activated regions. In the between-group analysis, clusters that passed the voxel-level uncorrected threshold of $\mathrm{p}<0.005$ and exceeded 20 voxels in size were considered activated regions.

AL-CL (investigating significantly activated regions when performing aesthetic judgment tasks compared to control judgment tasks for landscape images) and AF-CF (investigating significantly activated regions when performing aesthetic judgment tasks compared to control judgment tasks for fractal images) were selected as the contrast conditions. After conducting individual-level analyses, the within-group analysis was performed to verify regions that were significantly activated within each group. The between-group analysis was performed to compare differences in activated brain regions between the two groups. Moreover, to examine the correlation between the brain activity of significantly activated regions from the within-group analysis and the aesthetic judgment task scores of subjects in each group, a regression analysis was performed using SPM8 software.

The normality of the age, IQ, and psychological characteristics of the two groups was verified with Shapiro-Wilk tests, and the data were then compared with independent sample t-tests if they satisfied normality, or Mann-Whitney $U$ tests if they failed to satisfy normality. Gender and dominant hand were compared using chi-square analysis. Correlations between the variables were analyzed with Pearson's correlation coefficient analysis.

Statistical significance was set to $\mathrm{p}<0.05$ and SPSS version 21.0 (IBM Corp., Armonk, NY, USA) was used for the statistical analyses.

\section{RESULTS}

\section{Demographic and psychological characteristics (Table 1)}

There was no significant difference in age or gender between the ASD and control groups. There was also no significant difference in IQ and hand dominance between the two groups.

Upon comparing AQ, EQ, and SQ-R scores between the 
Table 1. Demographic and other characteristics of ASD group and HC group

\begin{tabular}{|c|c|c|c|c|}
\hline Characteristics & ASD group $(n=17)$ & HC group $(n=19)$ & tor U & $p$ \\
\hline Age (years)* & $17.65 \pm 1.80$ & $18.21 \pm 2.18$ & -0.841 & $0.406^{\dagger}$ \\
\hline $\mathrm{n}(\%)$ of $\mathrm{male}^{\ddagger}$ & $15(88.2)$ & $18(94.7)$ & $0.496^{\S}$ & 0.593 \\
\hline IQ & $96.81 \pm 11.31$ & $98.67 \pm 0.20$ & -0.518 & 0.608 \\
\hline Handedness ${ }^{\ddagger}$ & $15 / 2^{\| \prime}$ & $17 / 2^{\|}$ & $0.014^{\S}$ & 1.000 \\
\hline$A Q$ & $29.29 \pm 7.51$ & $16.84 \pm 5.77$ & 5.533 & $0.000^{\pi}$ \\
\hline$E Q$ & $25.12 \pm 12.64$ & $44.37 \pm 14.02$ & -4.333 & $0.000^{\pi}$ \\
\hline$S Q-R$ & $44.41 \pm 21.19$ & $46.53 \pm 20.59$ & -0.303 & 0.764 \\
\hline
\end{tabular}

Data are presented as mean \pm standard deviation unless otherwise indicated. ${ }^{*}$ comparison by Mann-Whitney test, ${ }^{\dagger}$ Mann-Whitney's $U$, ${ }^{\ddagger}$ comparison by chi-square test, ${ }^{\S} \chi^{2}$, "right-handed/ambidextrous, " $\mathrm{p}<0.001$. AQ: autism spectrum quotient, ASD: autism spectrum disorder, EQ: empathy quotient, HC: healthy control, IQ: intelligence quotient, SQ-R: systemizing quotient-revised

Table 2. Rating score and reaction time of ASD group and HC group

\begin{tabular}{|c|c|c|c|c|}
\hline Characteristics & ASD group $(n=17)$ & HC group $(n=19)$ & $t$ & $p$ \\
\hline \multicolumn{5}{|l|}{ Rating score } \\
\hline A-total & $2.40 \pm 0.51$ & $2.74 \pm 0.38$ & -2.278 & $0.029 *$ \\
\hline $\mathrm{AL}$ & $2.79 \pm 0.63$ & $2.95 \pm 0.35$ & -0.910 & 0.372 \\
\hline $\mathrm{CL}$ & $2.15 \pm 0.43$ & $2.21 \pm 0.34$ & -0.467 & 0.644 \\
\hline $\mathrm{AF}$ & $2.02 \pm 0.63$ & $2.53 \pm 0.69$ & -2.312 & $0.027^{*}$ \\
\hline $\mathrm{CF}$ & $2.10 \pm 0.35$ & $2.15 \pm 0.31$ & -0.439 & 0.663 \\
\hline \multicolumn{5}{|l|}{ Reaction time } \\
\hline A-total & $1849.64 \pm 358.05$ & $1924.70 \pm 218.55$ & -0.749 & 0.461 \\
\hline $\mathrm{AL}$ & $1798.69 \pm 504.63$ & $1974.02 \pm 380.83$ & -1.166 & 0.253 \\
\hline $\mathrm{CL}$ & $1939.05 \pm 347.74$ & $2038.12 \pm 384.59$ & -0.812 & 0.423 \\
\hline $\mathrm{AF}$ & $1909.08 \pm 446.45$ & $1876.08 \pm 405.50$ & -0.231 & 0.819 \\
\hline $\mathrm{CF}$ & $1773.80 \pm 340.65$ & $1836.24 \pm 301.52$ & -0.579 & 0.566 \\
\hline
\end{tabular}

Data are presented as mean \pm standard deviation unless otherwise indicated. ${ }^{*} p<0.05$. A-total: aesthetic rating of total images, AF: aesthetic-fractal, AL: aesthetic-landscape, ASD: autism spectrum disorder, CF: control-fractal, CL: control-landscape, HC: healthy control

two groups, the AQ score was significantly higher for the ASD group, at $29.29 \pm 7.51$, compared to $16.84 \pm 5.77$ for the control group ( $\mathrm{t}=5.533, \mathrm{p}=0.000)$. The EQ score was significantly higher for the control group, at $44.37 \pm 14.02$, compared to $25.12 \pm$ 12.64 for the ASD group $(\mathrm{t}=-4.333, \mathrm{p}=0.000)$. There was no significant difference in SQ-R scores between the two groups.

In the correlation analysis between the EQ, SQ-R, and AQ scores, there was a significant negative correlation between the EQ and AQ scores ( $\mathrm{r}=-0.792, \mathrm{p}=0.000)$. However, there was no significant correlation between the EQ and SQ-R scores, nor between the AQ and SQ-R scores.

\section{Scores and reaction times for the fMRI tasks (Table 2)}

During the aesthetic judgment task, the ASD group had an average score of $2.40 \pm 0.51$ while the control group had a score of $2.74 \pm 0.38$ for all images, hence the ASD group had a significantly lower score than the control group $(\mathrm{t}=-2.278, \mathrm{p}=0.029)$.

There was no significant difference in the aesthetic judgment task scores for the landscape images between the two groups, nor was there a significant difference in the control judgment task for the landscape images. There was also no signifi- cant difference in the control judgment task scores for fractal images. However, scores for the aesthetic judgment task for fractal images was $2.02 \pm 0.63$ for the ASD group and $2.53 \pm 0.69$ for the control group, hence the ASD group had a significantly lower score than the control group $(t=-2.312, p=0.027)$.

No significant difference was observed for reaction times between the two groups for all images. There was also no significant difference in reaction times between the two groups in terms of reaction time for each task involving landscape images and each task involving fractal images.

\section{Correlation between psychological characteristics and aesthetic judgment task scores}

An investigation was performed on the correlation between each psychological characteristic and the aesthetic judgment task scores for all subjects. The results showed a positive correlation between the EQ and the aesthetic judgment task score for landscape images $(\mathrm{r}=0.341, \mathrm{p}=0.042)$ and between the EQ and the aesthetic judgment task score for fractal images $(\mathrm{r}=0.339, \mathrm{p}=0.043)$. In addition, there was a positive correlation between the SQ-R and the aesthetic judgment 
task score for landscape images $(\mathrm{r}=0.364, \mathrm{p}=0.029)$. On the other hand, there was a negative correlation between the $\mathrm{AQ}$ and aesthetic judgment task score for fractal images ( $\mathrm{r}=$ $-0.377, \mathrm{p}=0.023)$.

\section{fMRI results}

\section{Results of the within-group analysis}

The brain regions showing significant activation in the control group under the AL-CL condition included the right anterior cingulate gyrus (BA 24), right medial frontal gyrus (BA 10), bilateral superior frontal gyrus (BA 6/8/9), right angular gyrus (BA 39), right supramarginal gyrus (BA 40), right posterior cingulate gyrus (BA 31), bilateral middle temporal gyrus (BA 19/21), left inferior semilunar lobule, and right fusiform gyrus (BA 20). In contrast, the brain regions showing significant activation in the ASD group under the AL-CL condition included the left superior frontal gyrus (BA 6/8), left parahippocampal gyrus (BA 36), left inferior temporal gyrus (BA 20), left superior temporal gyrus (BA 22), and the right anterior cingulate gyrus (BA 32).

Under the AF-CF condition, the control group showed significant brain activation in the right superior frontal gyrus (BA 6), bilateral inferior semilunar lobule, right inferior frontal gyrus (BA 45/47), left inferior frontal gyrus (BA 47), left superior frontal gyrus (BA 8), right middle temporal gyrus
(BA 21/39), and right superior temporal gyrus (BA 38). Under the AF-CF condition, the ASD group showed significant brain activation in the left anterior cingulate gyrus, right superior frontal gyrus (BA 8), bilateral middle temporal gyrus (BA 21), right fusiform gyrus (BA 20), right cingulate gyrus (BA 31), left angular gyrus (BA 39), and left superior frontal gyrus (BA 6/8).

\section{Results of the between-group analysis}

Under the AL-CL condition, the right superior frontal gyrus (BA 6/9) showed significantly more activation in the control group than the ASD group, while the left cingulate gyrus (BA 31), bilateral inferior semilunar lobule, left middle frontal gyrus (BA 6), left angular gyrus (BA 39), bilateral inferior parietal lobule (BA 40), right medial frontal gyrus (BA 6), right cerebellar tonsil, left insula (BA 13), and right precuneus (BA 7) showed significantly more activation in the ASD group than the control group (Table 3, Fig. 2).

Under the AF-CF condition, the left cingulate gyrus (BA 32), left superior frontal gyrus (BA 9), and left inferior semilunar lobule showed significantly more activation in the control group than the ASD group. On the other hand, the left claustrum, right amygdala, right middle temporal gyrus and inferior temporal gyrus (BA 37), left culmen, and left insula showed significantly more activation in the ASD group than the control group (Table 4, Fig. 3).

Table 3. Significantly activated areas when 'AL' condition was compared to 'CL' condition (AL-CL Condition)

\begin{tabular}{|c|c|c|c|c|c|c|c|}
\hline \multirow{2}{*}{ Region } & \multirow{2}{*}{ Side } & \multirow{2}{*}{ BA } & \multicolumn{3}{|c|}{ MNI coordinates } & \multirow{2}{*}{ Peak t-value } & \multirow{2}{*}{ Cluster size (voxels) } \\
\hline & & & $x$ & y & $\mathrm{z}$ & & \\
\hline \multicolumn{8}{|l|}{ HC group > ASD group } \\
\hline Superior frontal gyrus & Right & 6 & 8 & 32 & 60 & 3.93 & 37 \\
\hline Superior frontal gyrus & Right & 9 & 10 & 53 & 24 & 3.58 & 45 \\
\hline \multicolumn{8}{|l|}{ ASD group > HC group } \\
\hline Cingulate gyrus & Left & 31 & -16 & -44 & 28 & 3.7 & 54 \\
\hline Inferior semilunar lobule & Left & & -19 & -68 & -43 & 3.67 & 74 \\
\hline Inferior semilunar lobule & Left & & -14 & -65 & -36 & 2.86 & \\
\hline Middle frontal gyrus & Left & 6 & -26 & -4 & 47 & 3.59 & 75 \\
\hline Angular gyrus & Left & 39 & -35 & -72 & 33 & 3.51 & 353 \\
\hline Angular gyrus & Left & 39 & -31 & -57 & 33 & 3.32 & \\
\hline Inferior parietal lobule & Left & 40 & -42 & -54 & 42 & 2.77 & \\
\hline Medial frontal gyrus & Right & 6 & 13 & -14 & 54 & 3.11 & 21 \\
\hline Cerebellar tonsil & Right & & 27 & -58 & -33 & 3.03 & 54 \\
\hline Inferior parietal lobule & Right & 40 & 35 & -52 & 36 & 2.98 & 36 \\
\hline Cerebellar tonsil & Right & & 44 & 59 & -42 & 2.95 & 71 \\
\hline Inferior semi-lunar lobule & Right & & 42 & -67 & -41 & 2.86 & \\
\hline Insula & Left & 13 & -34 & 21 & 0 & 2.88 & 60 \\
\hline Precuneus & Right & 7 & 24 & -57 & -28 & 2.85 & 47 \\
\hline Precuneus & Right & 7 & 22 & -57 & 35 & 2.81 & \\
\hline
\end{tabular}

Thresholded at $\mathrm{p}<0.005$ uncorrected voxel level, extent threshold 20 voxels. AL: aesthetic-landscape, ASD: autism spectrum disorder, BA: Brodmann area, CL: control-landscape, HC: healthy control, MNI: Montreal Neuroimaing Institute 


\section{Results of the regression analysis (Fig. 4)}

In the results of the regression analysis between the aesthetic judgment task score and the degree of brain activation under the AL-CL condition, there was a significant positive correlation in the left anterior nucleus of the thalamus, left head of the caudate nucleus, and left dorsomedial nucleus of the thalamus for the control group. In contrast, there was a significant positive correlation in the bilateral anterior cingulate gyrus (BA 23/24), left superior temporal gyrus (BA 22/42), left middle frontal gyrus (BA 10), left inferior parietal lobule (BA 40), and right superior frontal gyrus (BA 9) for the ASD group.

In the regression analysis between the aesthetic judgment task score and the degree of brain activation under the AF-CF condition, there was a significant positive correlation in the left caudate nucleus, left superior frontal gyrus (BA 8), right cingulate gyrus (BA 23), and left middle frontal gyrus (BA 10/46) for the control group. Under the same condition, the
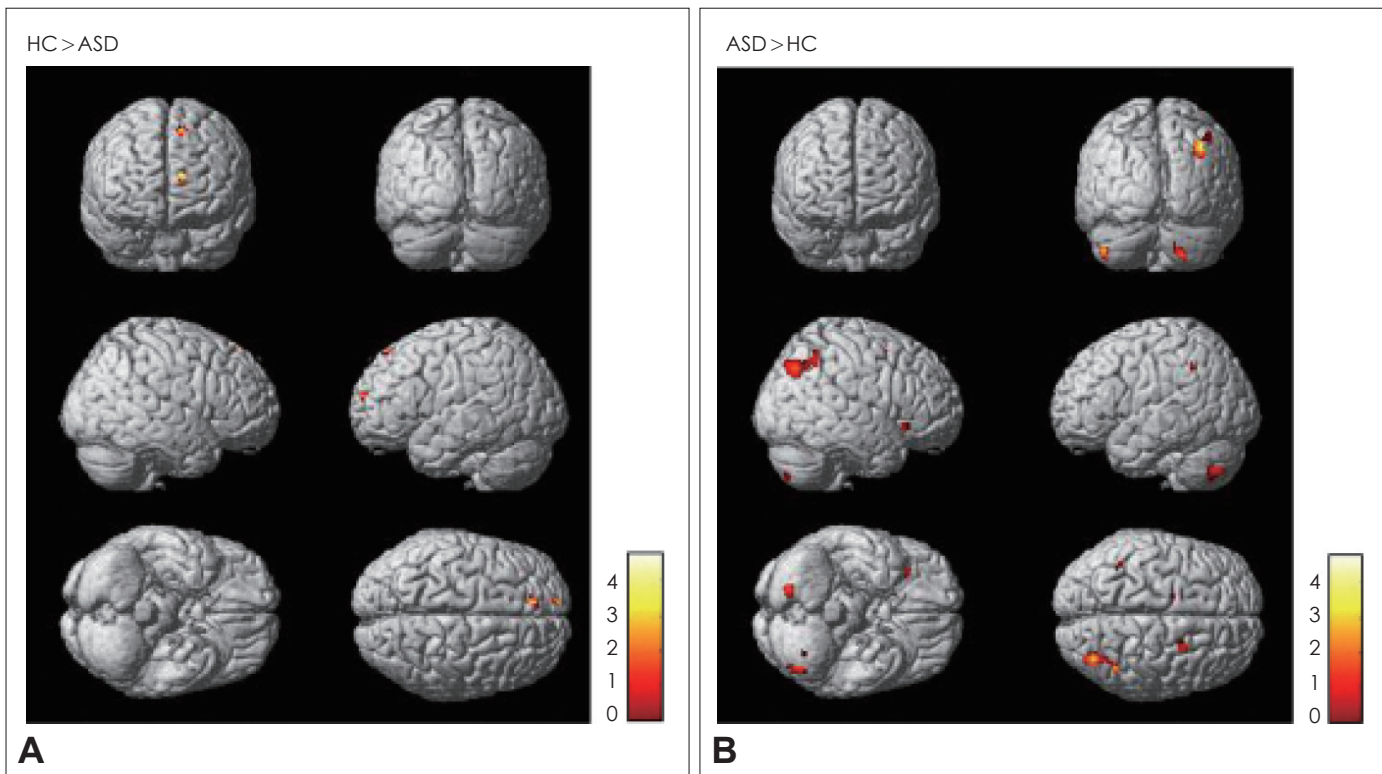

Fig. 2. Brain regions showing significant activation related on aesthetic judgement conditions with $A L-C L$ Contrast. $A$ : In $A L-C L$ contrast, HC group exhibited more activation in the right superior frontal gyrus (BA 6, 9) than ASD group. B: In the same contrast, ASD group showed relative hyperactivation in the left cingulate gyrus (BA 31), cerebellum, left middle frontal gyrus (BA 6), left angular gyrus (BA 39), bilateral inferior parietal lobule (BA 40), right medial frontal gyrus (BA 6), left insula (BA 13), right precuneus (BA 7) compared to HC group. AL: aesthetic-landscape, ASD: autism spectrum disorder, BA: Brodmann area, CL: control-landscape, Color bar: t-value, HC: healthy control.

Table 4. Significantly activated areas when 'AF' condition was compared to 'CF' condition (AF-CF condition)

\begin{tabular}{|c|c|c|c|c|c|c|c|}
\hline \multirow{2}{*}{ Region } & \multirow{2}{*}{ Side } & \multirow{2}{*}{ BA } & \multicolumn{3}{|c|}{ MNI coordinates } & \multirow{2}{*}{ Peak t-value } & \multirow{2}{*}{ Cluster size (voxels, } \\
\hline & & & $x$ & $y$ & $z$ & & \\
\hline \multicolumn{8}{|l|}{ HC group > ASD group } \\
\hline Cingulate gyrus & Left & 32 & -12 & 22 & 30 & 3.29 & 25 \\
\hline Superior frontal gyrus & Left & 9 & -14 & 44 & 32 & 3.21 & 38 \\
\hline Superior frontal gyrus & Left & 9 & -23 & 41 & 33 & 3.15 & \\
\hline Inferior semilunar lobule & Left & & -32 & -78 & -43 & 3.19 & 41 \\
\hline Inferior semilunar lobule & Left & & -38 & -72 & -43 & 3.12 & \\
\hline \multicolumn{8}{|l|}{ ASD group $>\mathrm{HC}$ group } \\
\hline Claustrum & Left & & -25 & 20 & 15 & 3.61 & 64 \\
\hline Amygdala & Right & & 33 & -7 & -21 & 3.55 & 50 \\
\hline Middle temporal gyrus & Right & 37 & 45 & -62 & 5 & 3.43 & 85 \\
\hline Inferior temporal gyrus & Right & 37 & 49 & -71 & 2 & 2.85 & \\
\hline Culmen & Right & & 21 & -29 & -23 & 3.21 & 36 \\
\hline Insula & Left & 13 & -42 & -2 & 3 & 2.9 & 28 \\
\hline
\end{tabular}

Thresholded at $\mathrm{p}<0.005$ uncorrected voxel level, extent threshold 20 voxels. AF: aesthetic-fractal, ASD: autism spectrum disorder, BA: Brodmann area, CF: control-fractal, HC: healthy control, MNI: Montreal Neuroimaing Institute 


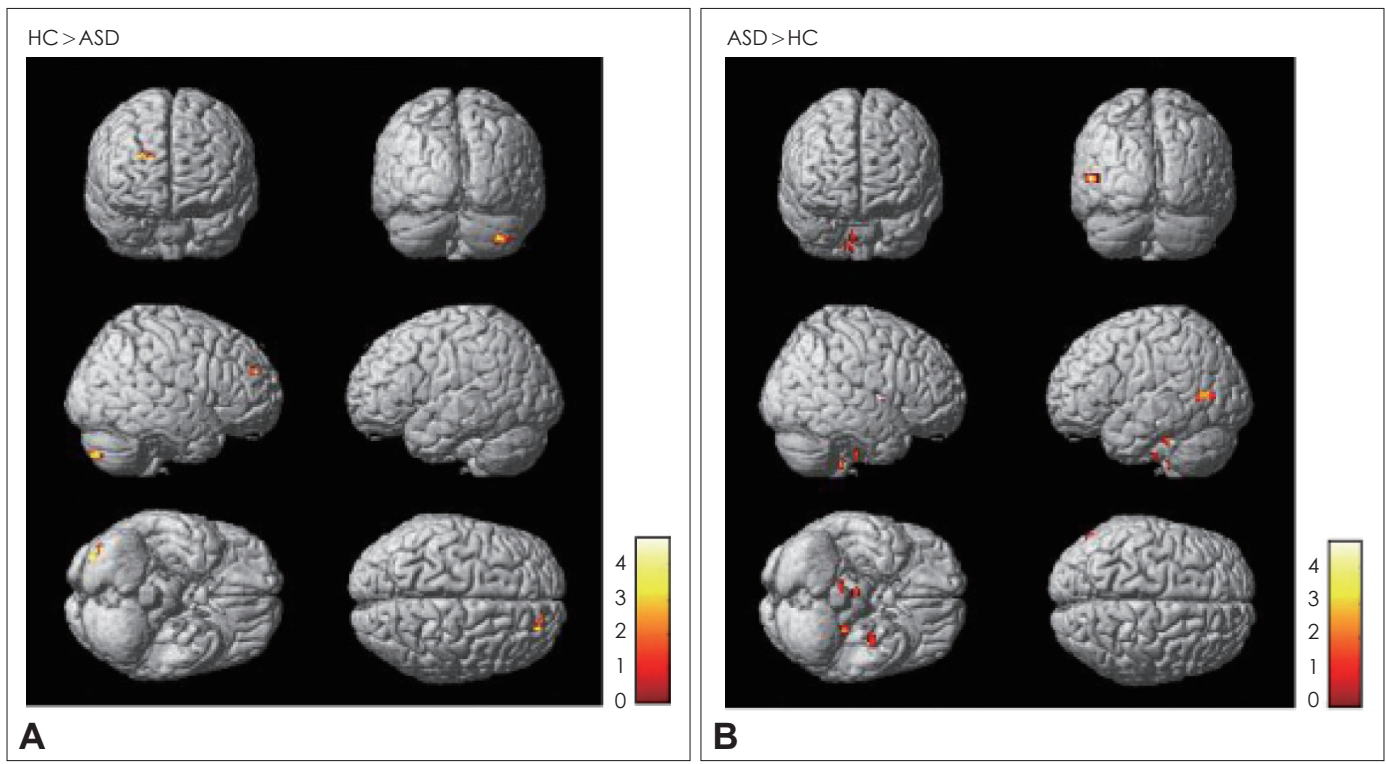

Fig. 3. Brain regions showing significant activation related on aesthetic judgement conditions with AF-CF contrast. A: In AF-CF contrast, HC group exhibited more activation in the left cingulate gyrus (BA 32), left superior frontal gyrus (BA 9), left inferior semilunar lobule of the cerebellum than ASD group. B: In the same contrast, ASD group showed relative hyperactivation in the left claustrum, right amygdala, right middle temporal gyrus (BA 37), right culmen of the cerebellum, left insula compared to HC group. AF: aesthetic-fractal, ASD: autism spectrum disorder, BA: Brodmann area, CF: control-fractal, Color bar: t-value, HC: healthy control.

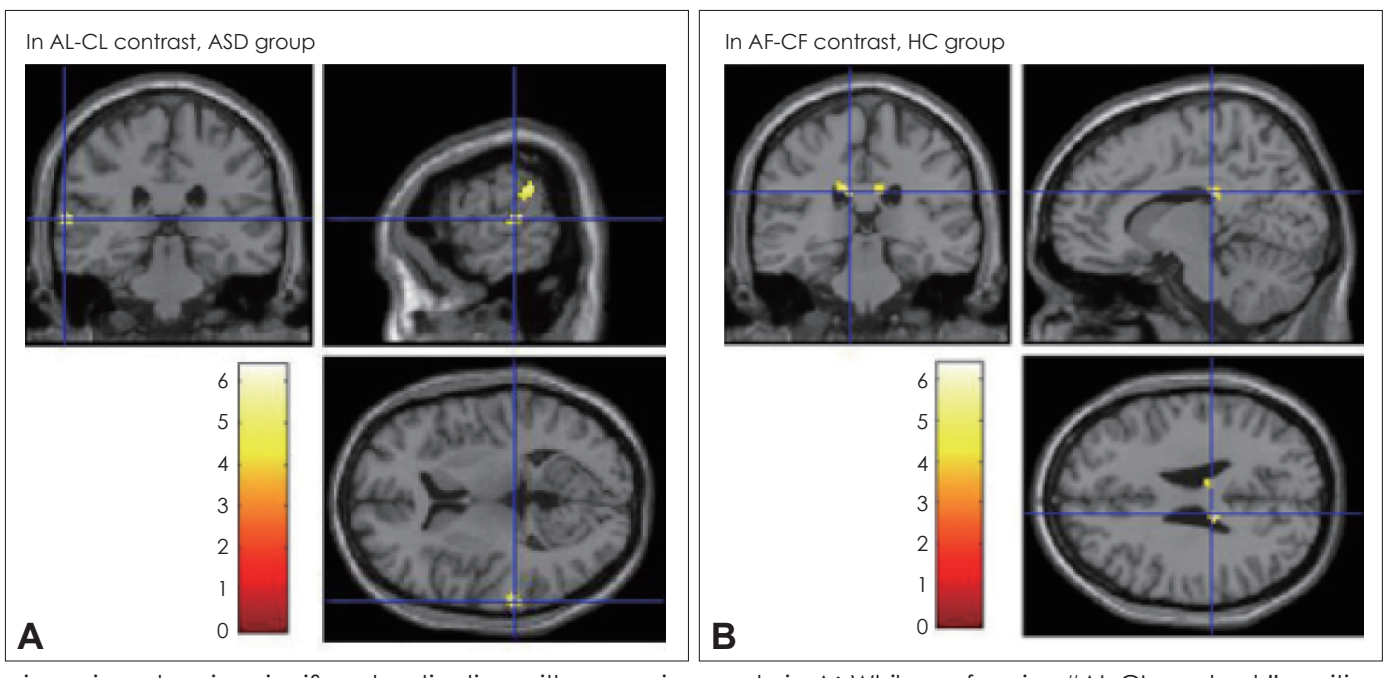

Fig. 4. Brain regions showing significant activation with regression analysis. A: While performing "AL-CL contrast," positive correlations were found in the ASD group between activity in various areas. The activated areas of left superior temporal gyrus and left inferior parietal lobule are seen. B: While performing "AF-CF contrast," positive correlations were found in the HC group between activity in various areas. The activated areas of left caudate and right cingulate gyrus are seen. AF: aesthetic-fractal, AL: aesthetic-landscape, ASD: autism spectrum disorder, BA: Brodmann area, CF: control-fractal, CL: control-landscape, HC: healthy control.

ASD group showed a significant positive correlation in the right anterior cingulate gyrus (BA 32), right middle frontal gyrus (BA 11), right superior temporal gyrus (BA 22), and left precentral gyrus (BA 6).

\section{DISCUSSION}

There was a significant difference in the EQ and AQ scores between the two groups, which is consistent with results from previous studies. However, the SQ-R score showed no significant difference between the two groups. Since the development of the SQ-R, many studies have reported that ASD patients showed significantly higher scores on this questionnaire, although some studies have reported no significant difference in the SQ-R score between ASD patients and normal individuals. ${ }^{25,26)}$ With respect to these results, Ghim et al. ${ }^{26)}$ proposed that a comparison on the relative differences between EQ and SQ-R score is more important than a comparison be- 
tween the SQ-R scores themselves. From the EQ, SQ-R, and AQ scores, the correlation analysis results only showed a significant negative correlation between EQ and AQ scores, implying that the EQ and $\mathrm{AQ}$ scores are the only ones that interact with each other. These results are consistent with those of Ghim et al. ${ }^{26)}$ In other words, higher autistic tendencies are associated with lower empathy.

It is important to note that the aesthetic judgment score of the ASD group was significantly lower than the control group in terms of the aesthetic judgment for all images. If the images were categorized, while there was no significant difference in the aesthetic judgment scores for landscape images between the two groups, the ASD group's aesthetic judgment score for the fractal images was significantly lower than the control group's score. Fractal properties are easily found in nature, but it is difficult to encounter fractal images for most people. ${ }^{24)}$ Therefore, these results imply that the ASD group felt that the fractal images were less familiar, and therefore less beautiful than did the control group.

In the correlation between the aesthetic judgment scores and the psychological characteristics for all subjects, the EQ score was positively correlated with the aesthetic judgment scores on both landscape images and fractal images. These results imply that an individual with higher empathic ability will judge an image to be more beautiful. In contrast, there was a negative correlation between the AQ and the aesthetic judgment score for fractal images. In other words, an individual with greater autistic tendencies is more likely to judge a fractal image as less beautiful.

The results of the within-group analysis showed that there was more brain activation in the frontal lobe, cingulate gyrus, superior temporal gyrus, and middle temporal gyrus for both the ASD group and the control group when making aesthetic judgments, compared to control judgments. These results were similar in both landscape images and fractal images.

Brain regions that are often reported to be related to aesthetic experiences in neuroaesthetic studies include the superior frontal area, which is related to laughing, positive emotions, and acquisition of aesthetic views by controlling priming towards the pragmatic view, ${ }^{11,27-29)}$ the cingulate gyrus, related to attention and emotional information processing, ${ }^{24,30)}$ and the middle temporal gyrus, which is related to semantic processing. ${ }^{10,24)}$

Therefore, such results from the within-group analysis support the conclusion that the aesthetic judgment tasks in this study properly activated the relevant brain regions.

The more notable result in the between-group analysis was that the ASD group showed significantly lower activation in the anterior region of the superior frontal gyrus (BA 9) compared to the control group, regardless of the type of stimu- lus. However, the ASD group showed predominantly greater activation in the angular gyrus (BA 39), inferior parietal lobule (BA 40), posterior region of middle/inferior temporal lobe, and insula (BA 13), when compared to the control group. In other words, when making aesthetic judgments, the ASD group had relatively less activation in the anterior areas of the brain, such as the dorsolateral prefrontal area, compared to normal individuals, while the posterior areas, such as the temporoparietal area, became relatively more active. These results imply that when the ASD group has aesthetic experiences, the dorsolateral prefrontal area that makes cognitive adjustments to control the pragmatic view and maintain the aesthetic view ${ }^{11,29)}$ is less activated than in normal individuals, while there is more activation in the temporoparietal area, which is related to visual analysis or visuospatial processing of objects.

The right superior frontal gyrus (BA 6/9), which was less activated in the ASD group than in the control group, has been reported to be related to laughing and positive reactions. ${ }^{27)}$ Therefore, the ASD group may have experienced less positive emotions during aesthetic judgments of images than the control group.

The angular gyrus (BA 39) and inferior parietal lobule (BA 40 ), which showed greater activation for the ASD group than the control group, have been reported to be connected to somatosensory spatial discrimination ${ }^{31)}$ and visuospatial processing. ${ }^{32)}$ The relative activation of the left insula (BA 13) in the ASD group is also important. Cognitive processing occurs in the left insula to assess appropriate context using affect regarding emotional experiences. ${ }^{11,30)}$ In addition, the insula has been shown to be activated when making objective judgments on the beauty of aesthetic stimuli. ${ }^{29)}$ Therefore, these results suggest that brain areas that are used during a physical or visuospatial analysis of stimuli during an aesthetic experience, or used to objectively judge the beauty of objects, are hyperactivated in a compensatory manner in ASD patients.

It is necessary to note that the amygdala and posterior regions of the middle/inferior temporal gyrus (BA 37) showed significantly more activation in the ASD group under the AF$\mathrm{CF}$ condition. The amygdala is associated with negative emotions, anxiety, and surprise. ${ }^{33)}$ The amygdala determines what kind of emotional reaction a person will make toward emotional information based on their emotional memory. The amygdala of ASD patients is hyperactive to stimuli that would be considered weak to normal individuals. ${ }^{34,35)}$ Some studies use this mechanism to explain the social phobia that is demonstrated by ASD patients. ${ }^{36)}$ Our study showed that the amygdala had significantly increased activation in the ASD group than the control group when making aesthetic judgments on fractal images, which implies that ASD patients experi- 
enced greater anxiety or surprise from fractal images than normal individuals. These negative emotions may have contributed to why the ASD group judged fractal images as being less beautiful.

Moreover, BA 37 includes the fusiform gyrus, which has functional connections with the amygdala. While it serves to recognize the facial information and affect of other people, it is also related to structure judgment regarding familiar objects $^{37)}$ or colors, and maintaining attention. ${ }^{38)}$ In previous studies, BA 37 was more activated for individuals with ASD than for normal individuals when tasks such as calculations were given, and greater activation of this area was correlated to greater task performance skills in the ASD group. ${ }^{39)}$ The fractal stimuli used in this study have mathematically consistent regularities, and such regularities induce higher concentration in the ASD group, which may have caused the increased activation of the BA 37 area.

In the results of the regression analysis, there was a significant positive correlation between the aesthetic judgment score and degree of activation of the caudate nucleus for both landscape images and fractal images in the control group. However, the ASD group did not produce the same result. Activation of the reward system in the brain during aesthetic experience is often reported, ${ }^{12,15)}$ but the results of this study imply that the reward system may be relatively less active in the ASD group than in normal individuals during an aesthetic experience. While there was a significant correlation between the aesthetic judgment score and degree of activation of the superior temporal gyrus (BA 22) for both landscape images and fractal images in the ASD group, no significant correlations were found in the control group. BA 22 pertains to Wernicke's area, which is related to processing auditory language ${ }^{40)}$ and semantic analysis, ${ }^{41)}$ and there have been reports of its association with processing non-linguistic auditory stimuli in the non-dominant hemisphere. ${ }^{42}$ The activation of this area related to auditory and language reception when making judgments on the beauty of visual stimuli appears to be a slightly unusual result.

The following are the limitations of this study. First, there were only a few subjects in each group. However, we made an effort to secure statistical significance by matching the IQ and age of each group. Second, the age of the subjects was limited to 15-22 years old, hence our study was unable to reflect differences that may appear among older or younger ASD patients and normal individuals. Third, this study was unable to use structured assessment tools such as the Autism Diagnostic Observation Schedule ${ }^{43}$ or the Autism Diagnostic Interview-Revised ${ }^{44)}$ for the ASD diagnosis. However, the ASD patients who participated in this study had all been diagnosed by psychiatrists who completed child and adoles- cent psychiatry fellowships, with many years of clinical experience, and the autistic characteristics of the ASD patients that were surveyed through the AQ were roughly twice as high as the control group. Fourth, all participants in this study had an IQ of 70 or higher. In $50-70 \%$ of individuals with ASD, the disorder is accompanied by intellectual disabilities, ${ }^{45)}$ which makes it difficult to apply the results of this study to all ASD patients. However, in order for subjects to properly understand and perform the fMRI tasks presented in this study, ASD patients with a certain level of cognitive function were required. Moreover, the majority of fMRI studies have been performed on subjects with an IQ of 70 or higher.

In spite of these limitations, this study holds significance in that it is the first study that has investigated differences in brain reactions when making aesthetic judgments between ASD patients and normal individuals. This study was able to verify that even typical ASD patients without savant skills showed differences in a degree of aesthetic judgments from normal individuals, and that their brain activation patterns when making aesthetic judgments also differ from normal individuals. Such results provide considerable implications for the social rehabilitation, designing of educational curricula, and establishing of art therapy processes for ASD patients. Going forward, more research on the aesthetic experience of ASD patients may be conducted using various other methods so that diverse research results can be applied to the treatment and rehabilitation of ASD patients.

\section{CONCLUSION}

During aesthetic judgments on artwork, the ASD group reported that the artwork was less beautiful than did the control group. The differences in aesthetic judgments of fractal images were particularly remarkable. Results of functional brain imaging showed that the dorsolateral prefrontal area was relatively less active in the ASD group when making aesthetic judgments, while the temporoparietal area and insula were hyperactive regardless of the type of the artwork. The amygdala and posterior region of the middle/inferior temporal gyrus were particularly more activated in the ASD group when making aesthetic judgments for fractal images. Such results suggest that the brain processing of ASD patients' aesthetic experiences may differ from that of normal individuals.

\section{Acknowledgments}

This work was supported by the National Research Foundation of Korea Grant funded by the Korean Government (NRF-2013S1A5 B6054557). 


\section{Conflicts of Interest}

The authors have no financial conflicts of interest.

\section{REFERENCES}

1) American Psychiatric Association. Diagnostic and statistical manual of mental disorders (DSM-5). 5th ed. Washington, DC: American Psychiatric Association;2013.

2) Bonoldi I, Emanuele E, Politi P. A piano composer with low-functioning severe autism. Acta Neuropsychiatr 2009;21:2-3.

3) Treffert DA. The savant syndrome: an extraordinary condition. a synopsis: past, present future. Philos Trans R Soc Lond B Biol Sci 2009;364:1351-1357.

4) Boso M, Emanuele E, Prestori F, Politi P, Barale F, D’Angelo E. Autism and genius: is there a link? the involvement of central brain loops and hypotheses for functional testing. Funct Neurol 2010;25: 15-20.

5) Son JW, Lee SB, Jung WH, Jee SH, Jung SH. What is neuroaesthetics?: a new paradigm in psychiatry. J Korean Neuropsychiatr Assoc 2013;52:3-16.

6) Frith U. Autism: explaining the enigma. Oxford: Blackwell;1989.

7) Happé F. Autism: cognitive deficit of cognitive style? Trends Cogn Sci 1999;3:216-222.

8) Wallace GL, Happé F, Giedd JN. A case study of a multiply talented savant with an autism spectrum disorder: neuropsychological functioning and brain morphometry. Philos Trans R Soc Lond B Biol Sci 2009;364:1425-1432.

9) Lee SH. 'Autistar', which dreams of a beautiful society with autistic people. [online] $2016 \mathrm{Apr}$ [cited 2017 May 1]. Available from URL: http://www.newstomato.com/readnews.aspx?no $=642088$.

10) Kim MM. The effect of the perceptual novelty on aesthetic experience: an fMRI study [dissertation]. Cheongju: Chungbuk Univ.;2013.

11) Cupchik GC, Vartanian O, Crawley A, Mikulis DJ. Viewing artworks: contributions of cognitive control and perceptual facilitation to aesthetic experience. Brain Cogn 2009;70:84-91.

12) Kawabata H, Zeki S. Neural correlates of beauty. J Neurophysiol 2004;91:1699-1705

13) Cunningham WA, Raye CL, Johnson MK. Implicit and explicit evaluation: fMRI correlates of valence, emotional intensity, and control int the processing of attitudes. J Cogn Neurosci 2004;16:1717-1729.

14) Zysset S, Huber O, Ferstl E, von Cramon DY. The anterior frontomedian cortex and evaluative judgment: an fMRI study. Neuroimage 2002;15:983-991.

15) Vartanian O, Goel V. Neuroanatomical correlates of aesthetic preference for paintings. Neuroreport 2004;15:893-897.

16) Kim CY. Neuroasethetics now-development and prospect. Korean J Cogn Biol Psychol 2015;27:341-365.

17) Kaufman J, Birmaher B, Brent D, Rao U, Flynn C, Moreci P, et al. Schedule for affective disorders and Schizophrenia for school-age children-present and lifetime version (K-SADS-PL): initial reliability and validity data. J Am Acad Child Adolesc Psychiatry 1997; 36:980-988.

18) Kim YS, Cheon KA, Kim BN, Chang SA, Yoo HJ, Kim JW, et al. The reliability and validity of Kiddie-Schedule for affective disorders and Schizophrenia-present and lifetime version- Korean version (K-SADS-PL-K). Yonsei Med J 2004;45:81-89.

19) Baron-Cohen S, Wheelwright S, Skinner R, Martin J, Clubley E. The autism-spectrum quotient (AQ): evidence from Asperger syndrome/high-functioning autism, males and females, scientists and mathematicians. J Autism Dev Disord 2001;31:5-17.

20) Park EH, Ghim HR, Cho KJ, Koo JS. Individual differences in empathizing and systemizing. Korean J Woman Psychol 2009;14: 269-286.

21) Baron-Cohen S, Wheelwright S. The empathy quotient: an investigation of adults with Asperger syndrome or high functioning au- tism, and normal sex differences. J Autism Dev Disord 2004;34:163175.

22) Baron-Cohen S. Autism: the empathizing-systemizing (E-S) theory. Ann N Y Acad Sci 2009;1156:68-80.

23) Wheelwright S, Baron-Cohen S, Goldenfeld N, Delaney J, Fine D, Smith R, et al. Predicting autism spectrum quotient (AQ) from the systemizing quotient-revised (SQ-R) and empathy quotient (EQ). Brain Res 2006;1079:47-56.

24) Lee SB, Jung WH, Son JW, Jo SW. Neural correlates of the aesthetic experience using the fractal images: an fMRI study. Sci Emot Sensib 2011;14:403-414.

25) Happé FG, Mansour H, Barrett P, Brown T, Abbott P, Charlton RA. Demographic and cognitive profile of individuals seeking a diagnosis of autism spectrum disorder in adulthood. J Autism Dev Disord 2016;46:3469-3480.

26) Ghim HR, Koo JS, Kim KM, Kim BN, Kim JW, Park M. Testing the extreme male brain theory of autism. Korean J Develop Psychol 2011;24:19-38.

27) Fried I, Wilson CL, MacDonald KA, Behnke EJ. Electric current stimulates laughter. Nature 1998;391:650.

28) Jacobsen T, Schubotz RI, Höfel L, Cremon DY. Brain correlates of aesthetic judgment of beauty. Neuroimage 2006;29:276-285.

29) Di Dio C, Macaluso E, Rizzolatti G. The golden beauty: brain response to classical and renaissance sculptures. PLoS One 2007;2: e1201.

30) Whalen PJ, Bush G, McNally RJ, Wilhelm S, McInerney SC, Jenike MA, et al. The emotional counting Stroop paradigm: a funtional magnetic rsonance imaging probe of the anterior cingulate affective division. Biol Psychiatry 1998;44:1219-1228.

31) Akatsuka K, Noguchi Y, Harada T, Sadato N, Kakigi R. Neural codes for somatosensory two-point discrimination in inferior parietal lobule: an fMRI study. Neuroimage 2008;40:852-858.

32) Köhler S, Kapur S, Moscovitch M, Winocur G, Houle S. Dissociation of pathways for object and spatial vision: a PET study in humans. Neuroreport 1995;6:1865-1868.

33) Davis M. The role of the amygdala in fear and anxiety. Annu Rev Neurosci 1992;15:353-375.

34) Ramachandran VS, Oberman LM. Broken mirrors: a theory of autism. Sci Am 2006;295:62-69.

35) Son JW, Ghim HR. Broken mirror or unbroken mirror?; an investigation for mirror neuron dysfunction of the autism spectrum disorder. J Korean Acad Child Adolesc Psychiatry 2013;24:109-123.

36) Kleinhans NM, Richards T, Weaver K, Johnson LC, Greenson J, Dawson G, et al. Association between amygdala response to emotional faces and social anxiety in autism spectrum disorders. Neuropsychologia 2010;48:3665-3670.

37) Kellenbach ML, Hovius M, Patterson K. A PET study of visual and semantic knowledge about objects. Cortex 2005;41:121-132.

38) Le TH, Pardo JV, Hu X. 4 T-fMRI study of nonspatial shifting of selective attention: cerebellar and parietal contributions. J Neurophysiol 1998;79:1535-1548.

39) Iuculano T, Rosenberg-Lee M, Supekar K, Lynch CJ, Khouzam A, Phillips J, et al. Brain organization underlying superior mathematical abilities in children with autism. Biol Psychiatry 2014;75:223230.

40) Ahmad Z, Balsamo LM, Sachs BC, Xu B, Gaillard WD. Auditory comprehension of language in young children: neural networks identified with fMRI. Neurology 2003;60:1598-1605.

41) McDermott KB, Petersen SE, Watson JM, Ojemann JG. A procedure for identifying regions preferentially activated by attention to semantic and phonological relations using functional magnetic resonance imaging. Neuropsychologia 2003;41:293-303.

42) Bernal B, Altman NR, Medina LS. Dissecting nonverbal auditory cortex asymmetry: an fMRI study. Int J Neurosci 2004;114:661680 . 
43) Lord C, Rutter M, Goode S, Heemsbergen J, Jordan H, Mawhood L, et al. Austism Diagnostic Observation Schedule: a standardized observation of communicative and social behavior. J Autism Dev Disord 1989;19:185-212.

44) Le Couteur A, Lord C, Rutter M. Autism Diagnostic Interview-Re- vised (ADI-R). Los Angeles, CA: Western Psychological Services; 2003.

45) Matson JL, Shoemaker M. Intellectual disability and its relationship to autism spectrum disorders. Res Dev Disabil 2009;30:1107-1114. 